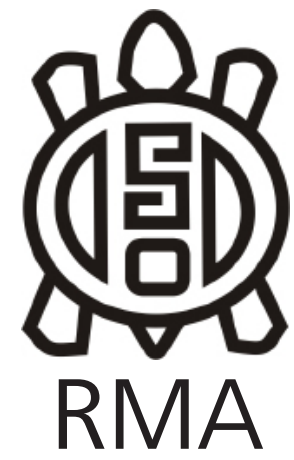

Dossier

\title{
Signos de nuestra época. Estado actual de la investigación arqueológica sobre las sociedades aldeanas tempranas en el Noroeste Argentino (2005-2019)
}

\author{
Signs of our epoch. Current state of archaeological research on early \\ village societies in Northwest Argentina (2005-2019).
}

Julián Salazar

*Instituto de Estudios Históricos, CONICET, Universidad Nacional de Córdoba, Argentina. E-mail: juliansalazar@ffyh.un.edu.ar

\begin{abstract}
Resumen
Estas notas valoran los aportes reunidos en el presente dossier, a la luz de un balance del estado actual de las investigaciones sobre el proceso de desarrollo, consolidación y desintegración de sociedades aldeanas tempranas del primer milenio d.C. en el Noroeste Argentino. A tal fin me concentro exclusivamente en la producción realizada en los últimos tres lustros y dada a conocer en cuatro de las principales publicaciones periódicas nacionales, así como en tres reuniones científicas específicas. En un contexto de ampliación de las posibilidades de practicar arqueología en nuestro país, que se registra desde 2002, se puede observar una multiplicación de proyectos en áreas anteriormente soslayadas, materializados desde instituciones dispersas en distintas provincias. Esta particularidad puede observarse también en una multiplicación de miradas y estudios específicos que han enriquecido nuestro conocimiento sobre las condiciones y estrategias sociales, así como de los mundos materiales, que dieron forma al modo de vida aldeano en este sector de nuestro país.
\end{abstract}

Palabras clave Noroeste argentino; Primer Milenio EC; Formativo; Investigaciones Arqueológicas.

\begin{abstract}
These notes assess the contributions joint in the present volume, in the light of the current state of research on the process of development, consolidation and disintegration of early village societies of the First Millennium A.D. in Northwest Argentina. To this end, I concentrate exclusively on the production carried out in the last three decades and published in four of the main national scientific journals, as well as in three specific meetings. In a context of growth of the possibilities of practicing archeology in our country, which has been recorded since 2002, we can observe a multiplication of projects in previously overlooked areas, materialized from institutions dispersed in various countries. This feature can also be observed in a multiplication of theoretical frameworks and specific studies that have enriched our knowledge about social conditions and strategies, as well as material worlds, which built the village way of life in this region of our country.
\end{abstract}

Keywords Northwest Argentina; First Millennium CE; Formative; Archaeological Researches.

El último cambio de siglo trajo consigo, al menos, tres publicaciones de síntesis sobre el Formativo en el Noroeste Argentino (en adelante, NOA). Tarragó (1999), en un tomo específico para el tema a nivel continental, y Albeck (2000) y Olivera (2001), en sendas compilaciones sobre arqueología argentina, mostraban, desde perspectivas un tanto disímiles, tres visiones sintéticas y bastante acabadas del periodo (o del tipo de adaptación, en el último caso), construidas en base a los conocimientos generados durante la segunda mitad del siglo $X X$, mayormente en algunos valles y quebradas clásicas y determinados bolsones puneños.

Los últimos tres lustros han significado, para la ciencia argentina, en general, y para la arqueología, en particular, un periodo de ampliación de posibilidades (aun con la retracción sufrida en el periodo 2015-2019), como el sostenimiento de una gran cantidad de proyectos colectivos, puestos laborales y becas doctorales que redundaron en la multiplicación de investigaciones generales y específicas, en zonas ya clásicas y en áreas inexploradas, aplicando metodologías tradicionales o diseñando herramientas novedosas, desde distintas líneas de pensamiento. Esta situación ha llevado a generar un estado de situación novedosa, en la cual conocemos tantas y tan variadas facetas de la vida y los procesos locales que sería realmente dificultoso llevar a buen puerto la tarea de construir una síntesis inteligible, coherente e integral, que pudiera dar cuenta de las primeras experiencias aldeanas en la región. La riqueza y diversidad 
de los estudios realizados sobre esta problemática hacen meritorio un balance de su estado actual, caracterizando a las personas que los efectúan, sus lugares de trabajo y/o formación, las áreas de estudio, los recortes temporales, las problemáticas y líneas de evidencias exploradas, así como las tendencias de las perspectivas teóricas.

Habiendo participado como comentarista del simposio que da origen a este dossier (ver Oliszewski y Spano en este volumen), analizo los trabajos aquí presentados a la luz de un relevamiento de aportes sobre la temática realizados entre 2005 y 2019, y publicados en cuatro revistas científicas específicas incluidas en el Núcleo Básico del Caicyt y editadas en distintas ciudades: Relaciones de la Sociedad Argentina de Antropología (Buenos Aires), Intersecciones en Antropología (Olavarría), Cuadernos (Jujuy) y Comechingonia (Córdoba). También considero tres reuniones específicas de la temática realizadas dentro de este periodo. El relevamiento de publicaciones se extiende a todas aquellas que hayan tomado como objeto de estudio algún aspecto de las sociedades aldeanas tempranas del NOA, así sean lecturas generales o estudios específicos, tomen una fracción temporal de sus trayectorias o incluyan a éstas en duraciones más largas. Con sociedades aldeanas tempranas hago referencia a aquellos grupos provisionales, improvisadores e innovadores que estructuran una gran variabilidad de dinámicas sociales, económicas, políticas, ambientales en el contexto en que en las secuencias regionales, por primera vez, las poblaciones comienzan a convivir de manera estable o recurrente en los mismos lugares (Bandy 2010). En términos cronológicos, este proceso se observa en el NOA en, lo que me atrevo a llamar, el largo primer milenio de la era cristiana, debido a que dichos procesos, que varían en distintas áreas de trabajo, pueden originarse unos siglos antes de la era y extenderse hasta momentos cercanos al siglo XIII dC.

Teniendo en cuenta estas consideraciones la muestra del análisis se constituye de 120 trabajos, 23 publicados en el libro surgido del Taller de Formativo de Tafí del Valle de 2012 (Korstanje et al. 2015), ocho en el número 20 de Andes (2009) dedicado al simposio de Formativo del XVI CNAA de Jujuy de 2007, 21 en Relaciones de la SAA, 23 en Intersecciones en Antropología, 15 en Cuadernos de la UNJu y 30 en Comechingonia (que incluyen ocho del N 211 , dedicado al simposio del primer milenio en el marco del XIX CNAA realizado en San Miguel de Tucumán en 2016).

\section{¿Quiénes somos, dónde trabajamos?}

El primer elemento destacable de este relevamiento es la cantidad de investigadores que han publicado trabajos sobre el primer milenio. El número asciende a 197, 116 mujeres y 81 hombres. Estos números muestran un signo de nuestra época, que es el crecimiento del protagonismo de las mujeres en la producción arqueológica, a pesar de las dificultades objetivas e históricas que presenta el campo disciplinario para ellas. En este aspecto, considerando solo trabajos de una autoría, se contabilizan $28(23,3 \%)$ firmados por una mujer y $9(7,5 \%)$, por un hombre. Entre los trabajos en coautoría, 46 (38,3\%) llevan primera autora, mientras que 37 (30,8\%), primer autor. Sin embargo, más allá de esta proporción exclusivamente cuantitativa sería interesante preguntarse por la relevancia e influencia de determinadas personas en el campo, valoración que queda en un ámbito exclusivamente subjetivo y puede tener otras muchas posibilidades. La influencia de los trabajos de Cristina Scattolin (2006) para lo que ella ha llamado el mundo precalchaquí, es incuestionable y puede verse sencillamente en ser una referencia obligada para quienes practicamos arqueología en el área y el periodo. En segundo lugar, el trabajo $y$, especialmente, las reflexiones de Alejandro Haber, han virado el eje de gran parte de las investigaciones posteriores a su tesis doctoral, defendida en 1999, pero publicada unos años después (Haber 2006). Más recientemente, la producción de Marcos Quesada, Norma Ratto y Alejandra Korstanje han marcado ciertos ejes de las investigaciones en sus áreas de estudio y, en parte, en las perspectivas aplicadas en otras.

La distribución geográfica de los lugares de trabajo resulta prometedora ya que, a pesar de la gran centralización remanente (el 30\% de las 197 autorías fijan su lugar de trabajo en alguna institución de Buenos Aires), los estudios sobre el mundo aldeano temprano se realizaron desde múltiples centros de investigación, dispersos en distintos puntos geográficos: Tucumán (25\%), Catamarca (11\%), Jujuy (8\%), Córdoba (5\%), La Plata (5\%), Salta (3,5\%), Mendoza (2\%), y La Rioja, Rio Cuarto, Mar del Plata, Entre Ríos y centros en el exterior (con porcentajes próximos al $1 \%$ cada uno). Es notable la importancia del sostenimiento de las carreras de Arqueología en Catamarca y Tucumán así como las tradiciones científicas y académicas de los distintos centros e instituciones de investigación de esas dos ciudades, lo que se traduce en que consideradas en conjunto superen al aporte de la CABA. Similar situación puede observarse en los trabajos reunidos en este dossier.

\section{¿Dónde y cuándo?}

Los tres lustros analizados han significado una verdadera ampliación y diversificación de las áreas de estudio cubiertas. La arqueología del Formativo estaba fuertemente centralizada en algunos valles intermontanos y pocas cuencas puneñas. Las narrativas planteaban incluso la existencia de una polarización histórica en la cual había núcleos de desarrollo y periferias dependientes que habrían incrementado su dependencia hasta niveles de integración regional, en la órbita Aguada por ejemplo (Núñez Regueiro y Tartusi 2002). En este registro, hemos detectado una buena cantidad de casos de las tres grandes zonas del NOA aún con un sesgo importante (Valles y Quebradas, 62\%; Puna 23\%, Piedemonte y Selva, 15\%). En este camino, la incorporación del aporte de Taboada (en este volumen) sobre la arqueología del río 
Salado, Santiago del Estero, previo al 1000 AP evidencia la necesidad de ampliar aún más las fronteras del alcance de estudios sobre contextos aldeanos, y del impacto potencial de estas ampliaciones en la comprensión de tipologías o estilos de materialidades que los caracterizaron, tanto en los ámbitos desconocidos como en los ámbitos con largas tradiciones de investigaciones. En otro caso, Juárez et al. (en este volumen) reúnen valiosa información sobre las prácticas alfareras en Tilcara durante el primer milenio, área muy conocida para momentos tardíos, pero casi imperceptible para el periodo en cuestión.

La multiplicación de proyectos en áreas previamente desconocidas o marginalizadas, sectores pedemontanos, cuencas tributarias de otras más conocidas o espacios intersticiales, empezó a horadar aquella visión de centroperiferia. Quesada et al. (2012), desde la vertiente oriental del Ancasti pudieron caracterizar una zona, previamente entendida como un sector de extracción de recursos dependiente de los centros Aguada, como un lugar habitado y cultivado, un espacio construido a partir de las relaciones de vecindad y que se constituía en sí mismo como un centro para sus habitantes. Esta sencilla pero profunda idea ha tenido una influencia sustantiva en los estudios actuales del primer milenio y es justamente la comprensión de la escala en la cual operan los procesos en el mundo aldeano temprano en el NOA. Lo local se ha reivindicado como la escala en la que se articulan las estructuras sociales y políticas de la época. Más allá de la existencia de lazos macrorregionales, redes de intercambio a larga distancia, modos de hacer replicados en gran escala, las dinámicas sociales y las lógicas de la práctica parecen construirse y reproducirse en lo local. Los ejes de la vida parecen ser definidos en los escenarios de la práctica cotidiana y en los entornos domésticos. Justamente la síntesis de estos dos aspectos, la intensa interacción con poblaciones alejadas y la centralidad del espacio cotidiano fue representada por Scattolin y su equipo (Scattolin et al. 2009) en sus investigaciones del espacio doméstico en Cardonal y Bordo Marcial, en el Valle del Cajón. Entre otros, el proyecto de la Quebrada de Los Corrales (Oliszewski 2011) ha tenido un rol protagónico en romper con la idea de áreas nucleares y periféricas observando la relevancia y originalidad de los procesos de una quebrada elevada (3100 msnm) y tributaria de la de Amaicha, a escasos kilómetros del fondo de un valle clásico para los estudios del primer milenio como es el de Tafí. Solo algunos frutos de ese proyecto se incluyen en Oliszewski y Di Lullo (en este volumen) y Gramajo Bühler y García Rosselló (en este volumen).

Como una contraparte de esta particularidad de los espacios y las lógicas de la práctica espacializada, los recortes temporales observados también muestran una configuración mucho más particular (acompañada de una creciente cantidad de fechados absolutos así como de un refinamiento de técnicas y procedimientos cronométricos) y local de lo que se esperaba en las secuencias tradicionales. Si bien algunos autores continúan encontrando productiva la referencia a categorías como Formativo temprano o Formativo tardío, los recortes temporales de los trabajos son muy variables y cada vez recurren más a una delimitación menos cargada y más estrictamente cronológica y abierta a variaciones. Esto muestra que los tempos de los desarrollos y cambios en las sociedades aldeanas también varían localmente (Franco en este volumen; Palamarczuk et al. en este volumen). Algunos procesos de las tierras altas puneñas muestran duraciones mucho más largas a las previamente imaginadas (Haber 2006), o evidencian perduraciones en algunos modos de hacer, y no en otros, que atraviesan a las periodificaciones (Orgaz et al. 2014).

\section{¿Cuáles son las problemáticas y las líneas de evidencia?}

El relevamiento de estudios realizado muestra una gran heterogeneidad de temáticas y líneas de evidencia, surgiendo 13 categorías en las cuales podemos agrupar a los 120 aportes: múltiples variables descriptivas (20); espacialidad (15); domesticidad (13); agricultura (6); funebria (18) ambiente (3); estratigrafía (1); cerámica (10); lítico (11); metalurgia (1); arqueofauna/malacología (7); arqueobotánica (8); Arte rupestre/representaciones plásticas (6); historiografía (1);

En primer lugar, podemos remarcar que una fuerte faceta de nuestra disciplina es la producción de datos y la ampliación de conocimiento empírico no demasiado problematizado, que resulta gran parte de las veces en un incremento muy positivo de evidencias sobre distintas regiones y procesos y, en efecto, el grupo más representado de trabajos tienen ese perfil (16\%).

Las modalidades en las que se constituyen los espacios residenciales y productivos son desde luego dos problemáticas relevantes de esta época, ambos fusionados en preguntas mayores sobre el paisaje. Ese parece ser un eje sobre el que todos los proyectos asientan sus preguntas y una lingua franca que permite tender diálogos compartidos entre distintos casos de estudios o incluso enfoques teóricos. El paisaje puede entenderse desde perspectivas más ambientales (Maldonado et al. 2011), más culturales (Cortés 2013), o relacionales e integradoras (Orgaz et al. 2014). En cualquier caso, como muestra el aporte de Palamarczuk et al. (en este volumen) el paisaje es analizado en términos dinámicos, como un palimpsesto donde se superponen una multiplicidad de materiales y tiempos, prácticas humanas y agentes ambientales cuyo entramado es partícipe de los procesos históricos y, en tanto tal, constituye una excelente vía para aproximarse al conocimiento de las condiciones y estrategias que definen el devenir de las sociedades aldeanas.

El entorno doméstico, su historia depositacional, sus modalidades constructivas, las actividades realizadas, la sintaxis espacial de su organización, la reproducción de habitus, la organización económica, entre otros, han 
sido terreno fértil para la reflexión en distintas escalas y, como adelantábamos, dichas reflexiones tuvieron una influencia importante en el campo de estudio (el $10 \%$ de los artículos considerados). Olisewski y Di Lullo (en este volumen) proponen que el alcance de un modo de construir y habitar viviendas permite interpretar la existencia de lazos identitarios compartidos en una zona que incluye quebradas altas de prepuna, valles intermontanos y cuencas pedemontanas. En este sentido, la participación de la configuración espacial en la reproducción de vínculos sociales y, a su vez, la de estos vínculos en la reproducción de esas configuraciones marca uno de los signos de nuestra época. Esa idea ha sido intensamente trabajada y moldeada en estudios de microhistorias que revisan la sucesión de eventos depositacionales en la configuración de viviendas. Palamarczuk et al. (en este volumen) muestran cómo este tipo de estudio de campo y análisis puede demostrar continuidades y cambios en las prácticas, reocupaciones de lugares y tendencias de larga duración.

Los espacios agrícolas también han sido reivindicados como ámbitos significativos y activos, superando una visión exclusivamente funcional de los mismos y ocupan un lugar creciente entre las investigaciones sobre el primer milenio (5\%). Su estudio, sobre todo intentando deconstruir amplios espacios productivos, cargados de infraestructura compleja, en los módulos de trabajo de personas, desde la escala efectiva en la cual se originaron, crecieron y entraron en desuso ha tenido también una relevante influencia en el modo de entender a las sociedades aldeanas y sobre todo aquellas en donde se observaban procesos de intensificación (Korstanje et al. 2015).

Sin dudas la arqueología de la muerte es otro componente significativo (15\%), incluyendo múltiples enfoques, desde la caracterización bioantropológica de los cuerpos inhumados, con la incorporación de la isotopía que ha dado maravillosos resultados en torno a la procedencia y movilidad de personas (López Campeny et al. 2014), los contextos materiales funerarios, hasta la relevancia de los cuerpos en la construcción de paisajes (Cortés 2013). La línea que atraviesa todos estos aportes es la relevancia de cuerpos de ancestros, o sus corporizaciones en formas materiales no humanas, para la construcción y reproducción de los entornos aldeanos, tanto en la reclamación de espacios y recursos, como en la incorporación de estructuras relacionales en la subjetividad de agentes en el contexto de entornos cotidianos.

La organización tecnológica, el manejo de materias primas, los modos de hacer y la circulación de distintas manufacturas también ha sido un tema recurrentemente abordado (16\%) con resultados reveladores. Si una ventana por la cual se había analizado predominantemente a las sociedades del primer milenio era la cerámica, en parte debido al relevante papel de dicha materialidad dentro de la arqueología normativa, la producción de los últimos tres lustros muestra una superación notoria de ese sesgo. Resulta muy alentador encontrar, por ejemplo, que los estudios sobre tecnología lítica (11, de los 120 relevados), tanto en términos de organización tecnológica como de manejo de recursos, supere en cantidad a aquellos destinados a la cerámica (10). Quizás uno de los primeros llamados en este camino haya sido efectuado por Elías y Escola (2007) y a partir de allí se haya generado una tendencia sostenida a dar importancia a los conjuntos artefactuales de roca tallada en ámbitos agroalfareros, lo que ha traído nuevos aportes sobre movilidad, manejo de los recursos locales y alóctonos, estrategias caza, modos de hacer y de reproducir las pautas tecnológicas. Las lecturas sobre cerámicas arqueológicas, por su parte, han avanzado notablemente y este dossier es un buen ejemplo de ello. Gramajo Bühler y García Rosselló (en este volumen) proponen un estudio traceológico para abordar las técnicas del modelado a mano, para introducirse en las tradiciones de los habitantes de la Quebrada de Los Corrales. Este tipo de estudios, permite observar persistencias de larga duración en determinados aspectos de las operaciones tecnológicas más allá de los cambios de los atributos superficiales y específicos. El trabajo de Franco (en este volumen) muestra también cómo la combinación de estudios experimentales, petrográficos y cristalográficos puede aportar datos bastante precisos sobre la procedencia y uso de arcillas. Sin embargo, su aporte más provocativo es poder llevar esto a la interpretación de las transformaciones y continuidades sobre las relaciones entre los habitantes de Anfama y las arcillas en un sitio multicomponente.

La relación de humanos y otros seres, especialmente animales y vegetales, ha sido una problemática en crecimiento (8\%) que ha explorado múltiples líneas: ampliación del conocimiento de especies incorporadas al mundo aldeano, maneras de entender el rol de esas entidades, la solapada comprensión de lo doméstico y lo silvestre, la variabilidad de manejos de animales y vegetales, la incorporación de no humanos en el mundo ritual e interacciones a larga distancia, entre otras. Esos intereses son plasmados en el meticuloso trabajo de síntesis de información arqueobotánica que ofrecen Petrucci y Spano (en este volumen) quienes, además de recoger una gran variedad de especies incorporadas a la vida cotidiana de los habitantes de Soria 2, en el valle Yocavil, interpretan las acciones y aspectos que conformaron la cotidianidad del grupo social que habitó un espacio doméstico, las cuales incluyen obtención, preparación y consumo de alimentos, hilado, confección de utensilios, ritual, entre otras.

\section{¿Cuáles son las perspectivas teóricas?}

Si bien en un influyente y provocativo análisis historiográfico (Politis 2003), la arqueología argentina (hasta los inicios 
del periodo de producción aquí considerado) había sido caracterizada como una práctica en la cual perduraban perspectivas anticuadas maquilladas superficialmente con los marcos teóricos de moda, considero que los estudios de los últimos años han tenido una interesante renovación teórica, que además tuvo su contraparte en la aplicación de metodologías y técnicas específicas que buscan construir evidencia empírica compatible con estos marcos. Aún en el contexto de la importancia empírica que caracteriza a la arqueología, expresada en que el $40 \%$ de los aportes registrados no expresan, explicitan, ni discuten ningún aspecto teórico, el $60 \%$ restante sí lo hace.

Por un lado, se observa una fuerte línea de pensamiento relacionada con lo que se ha dado en llamar arqueología de la complejidad o desigualdad social ( $24 \%$ de los casos), que podríamos definir como un marco cuyo núcleo duro abreva en algunas expectativas del neoevolucionismo, pero cuyo repertorio fue modificado con herramientas novedosas, especialmente la agencia. Este tipo de enfoques han sido aplicados fundamentalmente a casos incorporados dentro de las órbitas de influencia Aguada (Dantas 2014), tratando de explicar los modos en los cuales se constituyó, según algunos autores, una sociedad claramente marcada por la desigualdad social. García (en este volumen), por ejemplo, retoma la perspectiva de la complejidad para acercarse al registro de Azul Pampa, sintetizando buena cantidad de líneas de evidencia disponibles para la Quebrada de Inca Cueva en el bloque 3000-1000 AP.

El marco reflexivo que definitivamente ha marcado a estos tres lustros de arqueología sobre el mundo aldeano, son distintas versiones de arqueologías interpretativas, especialmente enfoques relacionales que aplican la teoría de la práctica de sociólogos como Pierre Bourdieu o Antony Giddens (Orgaz et al. 2014). La renovación que han implicado estas estructuras de pensamiento se observan en todos los ámbitos reflexivos y de construcción empírica y alcanzan al 34\% de la muestra analizada. La revalorización de lo local y de las recursivas relaciones entre prácticas sociales y materialidad que ha sido referida a lo largo de este trabajo se contextualizan justamente en este giro teórico. Sin embargo, la bajada de expectativas y categorías conceptuales construidas en otras disciplinas y diseñadas para analizar otro tipo de sociedades (v.g. modernas, contemporáneas), no ha sido siempre exitosa y aún requiere de mucho trabajo. Varias alternativas que incorporan enfoques más eclécticos, o marcos afincados en los llamados nuevos materialismos (Cortés 2013) presentan alternativas interesantes $(15,6 \%)$, algunas ya siendo exploradas en proyectos que están en curso y que sin dudas serán los protagonistas de los próximos años de investigaciones.

Por último, vale destacar la renovación a perspectivas tipológicas propuesta desde perspectivas ecológicoevolutivas. Incorporando categorías que pretenden dar cuenta de estrategias de individuos y la articulación de las mismas para dar forma a los procesos evolutivos de gran alcance y duración, se propusieron renovadoras maneras de entender la cultura material y los mecanismos selectivos por los cuales distintos tipos prosperan reproductivamente o se extinguen (Muscio 2013). Sin embargo, esta perspectiva no ha tenido aún demasiada aceptación más allá de un reducido grupo $(4,8 \%)$.

\section{¿La historia en migajas, signo de esta época, puede reensamblarse?}

Resta por preguntarnos si aún permanece algo de lo que alguna vez se llamó Formativo o Temprano, si hay algo que une a todas las investigaciones aquí comentadas y analizadas o si es hora de abandonar definitivamente estas reuniones temáticas y los esfuerzos integradores. En principio considero que es muy notorio que nos une, con las variaciones remarcadas, un entramado espacial y temporal en el cual se suceden una serie de procesos y experiencias compartidas. Más allá de la fuerza de lo local, en la mayoría de los casos estudiados, la cual se materializa en la relevancia de los ejes materiales de la vida doméstica existen múltiples evidencias de una continua y dinámica interacción, tema repetidamente referido en publicaciones clásicas que ha sido actualmente retomado tanto en aspectos relacionados al tráfico de bienes, como a los movimientos de personas (López Campeny et al. 2014) y sobre todo a amplios espacios donde circulan conocimientos y modalidades de hacer (Oliszewski y Di Lullo, en este volumen; Taboada, en este volumen). En segundo lugar, las discusiones entramadas en las tres reuniones aquí consideradas, demuestran que existe un espacio común que nos atrae el cual está constituido por distintas formas de configurar y disputar la vecindad, es decir la vivencia y experiencia compartida y continuada en los mismos lugares a través de múltiples generaciones, como una situación novedosa, frágil y versátil en el marco de estructuras sociales y políticas bastante abiertas, heterogéneas y descentralizadas (Delfino et al. 2009). El mundo material creado y creador de la vida aldeana es finalmente el vertebrador que, en calidad de practicantes de la disciplina de las cosas (Olsen et al. 2014), provoca que, en casi todos los casos, nos encontremos hablando de un estado de relaciones que nos resulta familiar: determinados modos de concebir materias primas y manejar su transformación (Franco en este volumen; Gramajo Bühler y García Rosselló en este volumen; Juárez et al. en este volumen), de construir casas, habitarlas o abandonarlas (Palamarczuk et al. en este volumen), de transitar y percibir paisajes, de marcarlos con cuerpos de ancestros (García en este volumen) o sus presentaciones líticas, de vincularse a animales o a vegetales (Petrucci y Spano en este volumen). Esas relaciones entre, otras muchas, son las que aún justifican seguir pensando y compartiendo pensamientos acerca de las sociedades aldeanas tempranas en el Noroeste Argentino.

Córdoba, 31 de diciembre de 2019. 


\section{Agradecimientos}

Es mi deseo agradecer a tod@s I@s colegas que han hecho arqueología sobre el Formativo o el Primer Milenio en estos 15 años y en especial a aquell@s que participaron con sus contribuciones en el simposio "Historias locales y signos de época (II)", en el XX CNAA celebrado en Córdoba en julio de 2019. Sus coordinadoras, Nurit y Romina, han sido grandes compañeras en todo este proceso. A l@s evaluador@s cuyas sugerencias han mejorado sensiblemente la primera versión de este escrito. Se agradece fundamentalmente al esfuerzo de la sociedad argentina, y a sus instituciones, que han posibilitado hacer ciencia y, esperamos, lo seguirán haciendo.

\section{Bibliografía}

Albeck, M. E. (2000). La vida agraria en los Andes del Sur. En Tarragó, M. N. (Ed.), Nueva Historia Argentina Tomo I (pp. 187-228). Buenos Aires: Sudamericana.

Bandy, M. S. (2010). Population Growth, Village fisioning and Alternative Early village trajectories. In Bandy, M. S. y Fox, J. J. R. (Eds.), Becoming villagers: comparing early village societies (pp. 19-36). Tucson: The University of Arizona Press.

Cortés, L. (2013). A través del paisaje, a través de los cuerpos. Contextos funerarios del sur del valle del cajón (noroeste argentino, 6000-1300 años ap). Relaciones de la Sociedad Argentina de Antropología, 38(2), 293-319.

Dantas, M. (2014). El rol de los animales en contextos sociales no igualitarios: El caso del valle de Ambato, Catamarca, Argentina. Relaciones de la Sociedad Argentina de Antropología, 39 (1): 57-78.

Delfino, D. D., Espiro, V. E., y Díaz, A. (2009). Modos de vida situados: el formativo en Laguna Blanca. Andes, 20, 111-134.

Elías, A. M., y Escola, P. (2007). Estrategias de aprovechamiento y uso de recursos liticos en sociedades agrícolas-pastoriles de la puna meridional Argentina. Cuadernos de La Facultad de Humanidades y Ciencias Sociales - Universidad Nacional de Jujuy, 32, 111-133.

Haber, A. F. (2006). Una arqueología de los oasis puneños. Córdoba: Sarmiento Editor.

Korstanje, M. A., Lazzari, M., Basile, M., Bugliani, F., Lema, V., Pereyra, L., y Quesada, M. (2015). Crónicas materiales precolombinas. Buenos Aires: SAA

Korstanje, A., Quesada, M., Franco Salvi, V., Lema, V., y Maloberti, M. (2015). Gente, tierra, agua y cultivos: Ios primeros paisajes agrarios del Noroeste Argentino. En Korstanje, M., Lazzari, M., Basile, M., Bugliani, M. F., Lema, V., Pereyra Domingorena, L. y Quesada, M. (Eds.), Crónicas materiales precolombinas, Arqueología de los primeros poblados del Noroeste argentino, (pp. 721-749). Buenos Aires: SAA.
López Campeny, S. M. L., Romano, A. S., Rodríguez, M. F., Martel, Á. R., y Corbalán, M. H. (2014). De aquí y de allá: Análisis integral de un contexto funerario. Vínculos e interacciones sociales entre Puna meridional y Tierras Bajas orientales. Intersecciones en Antropología, 15(1), 201-218.

Maldonado, M., Neder, L., Roldán, J., y Sampietro Vattuone, M. (2011). Caracterización geoambiental y cultural del período formativo en Selvas Occidentales Meridionales: sitio "Horco Molle" (Dpto. Yerba Buena, Tucumán). Comechingonia, 14, 115-131.

Muscio, H. (2013). El registro arqueológico de la quebrada de Urcuro, puna de Salta, Argentina, en una perspectiva evolucionista. Intersecciones en Antropología, 14 (1): 83-92.

Núñez Regueiro, V., y Tartusi, M. R. A. (2002). Aguada y el proceso de integración regional. Estudios Atacameños, 24, 9-19.

Olivera, D. (2001). Sociedades agro-pastoriles tempranas: el Formativo Inferior del Noroeste Argentino. En Berberián, E. y Nielsen, A. E. (Eds.), Historia Argentina Prehispánica. (pp. 83-126). Córdoba: Brujas.

Oliszewski, N. (2011). Ocupaciones prehispánicas en la quebrada de Los Corrales, El Infiernillo, Tucumán (ca. 2500-600 AP). Comechingonia. Revista de Arqueología, $14,155-172$.

Olsen, B., Shanks, M., Webmoor, T., y Witmore, C. L. (2012). Archaeology: the discipline of things. Berkeley: University of California Press.

Orgaz, M., Ratto, N. y Coll, L. (2014). Aportes para la construcción de los paisajes agrícolas en la región de Fiambalá, Tinogasta, Catamarca: Nuevas Evidencias. Relaciones de la Sociedad Argentina de Antropología, 39(1), 79-94.

Politis, G. G. (2003). The Theoretical Landscape and the Methodological Development of Archaeology in Latin America. American Antiquity, 68(2), 245-272.

Quesada, M. N., Gastaldi, M. R., y Granizo, G. M. (2012). Construcciones de periferias y producción de lo local en las cumbres de El Alto-Ancasti. Relaciones de La Sociedad Argentina de Antropología, 37(2), 435-456.

Scattolin, M. C. (2006). Contornos y confines del universo iconográfico precalchaquí del valle de Santa María. Estudios Atacameños, 32, 119-139.

Scattolin, M. C., Bugliani, M. F., Cortés, L. I., Calo, C. M., Domingorena Pereyra, L., e lzeta, A. D. (2009). Pequeños mundos: hábitat, maneras de hacer y afinidades en aldeas del valle del Cajón, Catamarca. Relaciones de La Sociedad Argentina de Antropología, 34, 251-274.

Tarragó, M. N. (1999). El Formativo y el surgimiento de la complejidad social en el Noroeste argentino. En Ledergerber-Crespo, P. (Ed.), Formativo Sudamericano: una reevaluación (pp. 302-307). Quito: Abya-Yala. 